\title{
Editorial: The Post-Exome Era
}

dftation: Ohadi, M. The post-exome era. Iranian Rehabilitation Journal. 2016; 14(1): 3-4. http://dx.crossref.org/10.15412/J.IRJ.08140101

dol: : http://dx.crossref.org/10.15412/J.IRJ.08140101

The Iranian Rehabilitation Journal (IRJ) invites research papers on the genetic basis of single gene and complex disorders. This vastly dynamic branch of science will complement the multidisciplinary wealth of expertise in the fields of social welfare and rehabilitation. The past few years have witnessed outstanding research projects on the genetic causes of numerous debilitating disorders, such as intellectual disability, deafness, and blindness. Those achievements are largely indebted to the next generation exome sequencing technologies. Exome sequencing detects variants in coding exons, and can expand the target to include untranslated regions (UTRs) and microRNA to present a more comprehensive view of gene regulation. Whole-exome sequencing (WES) determines variations of all coding regions, or exons, of known genes. Once beyond imagination and reach, WES promises to revolutionize the perspective of a wide range of applications, including population genetics, medical genetics, and cancer studies.

The post-exome era belongs to the regulatory regions (e.g. promoters/enhancers), in which short tandem repeats (STRs or microsatellites) are of unique nature, as they span several DNA base pairs (bp) (and as a result can potentially recruit larger numbers of regulatory factors in comparison with single nucleotide substitutions), are highly mutable, and can expand or contract. STRs are highly polymorphic sequences consisting of subunits of 1-6 bp, that have extremely high mutation rates, and underlie substantial phenotypic variation at the inter-, and intra-species levels. It is, therefore, conceivable that the high mutation rate of STRs in functional genomic regions may facilitate evolutionary adaptation. It may be hypothesized that STRs that reach exceptional length in humans may be of selective advantage to this species.

The available genome-scale reports of genes that contain such STRs should pave the way for testing the above hypothesis. The emerging comparative and functional analyses of a number of those STRs support adaptive evolutionary patterns for expansion or contraction. Along that notion, it is reasonable to speculate that "human-specific" STRs may be linked to characteristics or disorders that are specific to humans. For example, the human-specific characteristic, cognition, and the ubiquitous compromise of this characteristic in several neuropsychiatric disorders, including schizophrenia, bipolar disorder, and Alzheimer's disease, may be linked to STRs that are specific to humans or have reached exceptional length in this species. To this end, a number of research projects have resulted in the proliferation of findings that support a link between STRs and human evolution. With the development of more efficient technologies for accurate and fast sequencing of these highly polymorphic and mutable motifs, STRs can bring the field of molecular genetics into a new era, which may transform the available knowledge in quantitative traits and complex disorders.

\footnotetext{
* Corresponding Author: Mina Ohadi, $M D, P h D$

Address: Genetic Research Center, University of Social Welfare and Rehabilitation Sciences, Kodakyar Ave., Daneshjoo Blvd., Evin, Tehran, Iran. Tel: +98 (21) 22180138

E-mail: ohadi.mina@yahoo.com
} 
